\title{
Supplementary Material for: Spatial risk mapping for rare disease with hidden Markov fields and variational EM
}

\author{
F. Forbes, M. Charras-Garrido, L. Azizi, S. Doyle, D. Abrial
}

\section{Introduction}

This paper contains supplementary material for the article entitled Spatial risk mapping for rare disease with hidden Markov fields and variational EM. In the latter work, we considered partition models for disease mapping. These models involve the introduction of a finite number of risk levels and allocation variables to assign each area under study to one of these levels. Spatial dependencies are then taken into account by modeling the allocation variables as a discrete state-space Markov random field (MRF). For this MRF term, we proposed a general formulation that is able to encode more complex interactions than the standard Potts model.

More specifically, to draw interpretable maps, with clearly delimited zones, we recast the disease mapping issue into a clustering task. Based on count data for a rare phenomenon observed in a predefined set $S$ of $N$ areas (e.g. geographical regions), the goal is to assign to each region a risk level among a finite set of $K$ possible levels $\left\{\lambda_{1}, \ldots, \lambda_{K}\right\}$ when these risk levels are themselves unknown and need to be estimated. In each area, two values are available, the number $y_{i}(i \in S=\{1, \ldots, N\})$ of observed cases of the given disease and the population size $n_{i}$. A common assumption is that for an area indexed by $i \in S$, the number of cases $y_{i}$ is a realization of a Poisson distribution whose parameter depends on the risk level assigned to the area. It is then convenient to consider the risk assignment for area $i$ as $z_{i}$ in a set of $K$-dimensional indicator vectors $\mathcal{L}=\left\{e_{1}, \ldots, e_{K}\right\}$ where each $e_{k}$ has all its components set to 0 except the $k$ th which is 1 .

Therefore, the data is naturally divided into observed count data $\mathbf{y}=\left\{y_{1}, \ldots, y_{N}\right\}$ and unobserved or missing membership data $\mathbf{z}=\left\{z_{1}, \ldots, z_{N}\right\}$. The latter are considered as random variables denoted by $\mathbf{Z}=\left\{Z_{1}, \ldots, Z_{N}\right\}$. The dependencies between neighboring $Z_{i}$ 's are then modelled by further assuming that the joint distribution of $\left\{Z_{1}, \ldots, Z_{N}\right\}$ is a discrete MRF on the graph connecting contiguous locations (i.e, regions $i$ and $j$ are neighbors if they are spatially contiguous):

$$
P(\mathbf{z} ; \beta)=W(\beta)^{-1} \exp (-H(\mathbf{z} ; \beta))
$$

where $\beta$ is a set of parameters, $W(\beta)$ is a normalizing constant and $H$ is a function restricted to pair-wise interactions, $H(\mathbf{z} ; \beta)=-\sum_{i \in S} z_{i}^{t} \alpha-\sum_{\substack{i, j \\ i \sim j}} z_{i}^{t} \mathbb{B} z_{j}$, where we write $z_{i}^{t}$ for the transpose 
of vector $z_{i}$ and $i \sim j$ when areas $i$ and $j$ are neighbors. The set of parameters $\beta$ consists of two sets $\beta=(\alpha, \mathbb{B})$. Parameter $\alpha$ is a $K$-dimensional vector which acts as weights for the different values of $z_{i}$. When $\alpha$ is zero, no risk level is favored, i.e. for a given area $i$, if no information on the neighboring areas is available, then all risk levels have the same probability. Then, $\mathbb{B}$ is a $K \times K$ matrix that encodes interactions between the different classes. If in addition to a null $\alpha, \mathbb{B}=b \times I_{K}$ where $b$ is a real scalar and $I_{K}$ is the $K \times K$ identity matrix, parameters $\beta$ reduce to a single scalar interaction parameter $b$ and we get the Potts model traditionally used for image segmentation. In practice, it may be more appropriate, from a disease mapping point of view, to encode higher penalties when the risk levels are further apart. We propose to use for $\mathbb{B}$ a matrix with three non zero diagonals defined for some positive real value $b$ by:

$$
\begin{aligned}
\mathbb{B}(k, k) & =b \quad \text { for all } k=1, \ldots, K \\
\mathbb{B}(k, l) & =b / 2 \text { for all }(k, l) \text { such that }|k-l|=1 \\
\mathbb{B}(k, l) & =0 \text { otherwise. }
\end{aligned}
$$

This is the simplest non standard $\mathbb{B}$ structure that can encode smooth variations in the risk level.

For the model to be fully defined, the observation model needs then also to be specified. The class dependent distribution is usually a standard distribution, typically in rare disease mapping, a Poisson distribution:

$$
P\left(Y_{i}=y_{i} \mid Z_{i}=z_{i} ; \lambda\right)=\mathcal{P}\left(y_{i} ; n_{i} z_{i}^{t} \lambda\right)=\exp \left(-n_{i} z_{i}^{t} \lambda\right) \frac{\left(n_{i} z_{i}^{t} \lambda\right)^{y_{i}}}{y_{i} !}
$$

where $n_{i}$ is the population size in area $i$ and $z_{i}^{t} \lambda$ with $\lambda=\left[\lambda_{1}, \ldots, \lambda_{K}\right]^{t}$ is a vectorial notation that indicates the dependence on the specific value of $z_{i}$ which determines the risk level.

For the distribution of the observed variables $\mathbf{y}$ given the classification $\mathbf{z}$, the usual conditional independence assumption leads to: $P(\mathbf{Y}=\mathbf{y} \mid \mathbf{Z}=\mathbf{z} ; \lambda)=\prod_{i \in S} \mathcal{P}\left(y_{i} ; n_{i} z_{i}^{t} \lambda\right)$. It follows that the conditional probability of the hidden field $\mathbf{z}$ given the observed field $\mathbf{y}$ is

$$
P(\mathbf{z} \mid \mathbf{y} ; \lambda, \beta)=W(\beta)^{-1} \exp \left(-H(\mathbf{z} ; \beta)+\sum_{i \in S} \log \mathcal{P}\left(y_{i} ; n_{i} z_{i}^{t} \lambda\right)\right) .
$$

The parameters of this model are then denoted by $\Psi=(\lambda, \alpha, \mathbb{B})$.

The question of interest is to recover the unknown assignment map $\mathbf{z}$. To do so, we consider a Maximum Posterior Marginal (MPM) principle consisting of assigning each region $i$ to the class $e_{k}$ that maximizes $P\left(Z_{i}=e_{k} \mid \mathbf{y} ; \boldsymbol{\Psi}\right)$. In this paper, to deal with the missing data and the spatial dependence structure, we use the EM algorithm [2] with some of the approximations presented in [1] based on the mean field principle.

Section A provides more details about the EM and mean field approximation used in the paper. We then addressed the issue of initializing such an EM procedure and Section B provides some details about the proposed initialization strategy. In Section $\mathbf{C}$ are given some additional observations using simulated data while Section D provides additional tests made on real data for the Bovine Spongiform Encephalopathy epidemic. 


\section{A Estimating disease maps using variational EM}

\section{A.1 A tractable EM variant}

The difficulty with MRF models is that the computation of $P(\mathbf{z} ; \beta)$ is not possible due to the normalizing constant $W(\beta)$ which involves $K^{N}$ terms and is intractable except in some trivial cases. In contrast the conditional probability $p\left(z_{i} \mid \mathbf{z}_{\mathcal{N}(i)} ; \beta\right)$, where $\mathbf{z}_{\mathcal{N}(i)}$ denotes the values $\left\{z_{j}, j \in \mathcal{N}(i)\right\}$ associated to the set $\mathcal{N}(i)$ of neighbors of $i$, is easily computed using the following formula:

$$
P\left(z_{i} \mid \mathbf{z}_{\mathcal{N}(i)} ; \beta\right)=\frac{\exp \left(z_{i}^{t}\left(\alpha+\mathbb{B} \sum_{j \in \mathcal{N}(i)} z_{j}\right)\right)}{\sum_{k=1}^{K} \exp \left(\alpha(k)+e_{k}^{t} \mathbb{B} \sum_{j \in \mathcal{N}(i)} z_{j}\right)} .
$$

More details are given below about the general form of the Mean Field like EM procedures introduced in [1] and used in the paper. Informally, the mean field approach consists of approximating the intractable probabilities by neglecting fluctuations from the mean in the neighborhood of each region $i$. This is obtained by assuming the neighboring $z_{j}$ for $j$ in $\mathcal{N}(i)$, fixed to their mean values. More generally, we talk about mean field-like approximations when the value $z_{i}$ does not depend on the values $z_{j}$ for $j \neq i$ which are all set to constants (not necessarily to the means) independently of the value of $z_{i}$. These constant values denoted by $\tilde{z}=\left\{\tilde{z}_{1}, \ldots, \tilde{z}_{N}\right\}$ are not arbitrary but satisfy some appropriate consistency conditions [see 1]. It follows that $P\left(z_{i} \mid \mathbf{y} ; \mathbf{\Psi}\right)$ is approximated by $P\left(z_{i} \mid \mathbf{y}, \tilde{z}_{\mathcal{N}(i)} ; \mathbf{\Psi}\right)$ which is proportional to:

$$
\mathcal{P}\left(y_{i} ; n_{i} z_{i}^{t} \lambda\right) P\left(z_{i} \mid \tilde{z}_{\mathcal{N}(i)} ; \beta\right) \propto \mathcal{P}\left(y_{i} ; n_{i} z_{i}^{t} \lambda\right) \exp \left(z_{i}^{t}\left(\alpha+\mathbb{B} \sum_{j \in \mathcal{N}(i)} \tilde{z}_{j}\right)\right) .
$$

The normalizing constant is not specified but its computation is not an issue as it involves only a sum over $K$ terms. Then, for all $j \in \mathcal{N}(i), P\left(z_{i}, z_{j} \mid \mathbf{y} ; \mathbf{\Psi}\right)$ is approximated by $P\left(z_{i} \mid \mathbf{y}, \tilde{z}_{\mathcal{N}(i)} ; \mathbf{\Psi}\right) P\left(z_{j} \mid \mathbf{y}, \tilde{z}_{\mathcal{N}(j)} ; \mathbf{\Psi}\right)$. Both approximations are easy to compute.

In particular the so-called mean field and simulated field algorithms correspond to two different ways of creating $\tilde{\mathbf{z}}$. The mean field algorithm consists of updating the $\tilde{z}_{i}^{\left({ }^{(q)}\right.}$ 's by setting, for all $i=1, \ldots, N, \tilde{z}_{i}^{(q)}$ to the mean of distribution $P\left(z_{i} \mid \mathbf{y}, \tilde{z}_{\mathcal{N}(i)}^{(q)} ; \Psi^{(q-1)}\right)$. Note that as $z_{i}$ is an indicator vector, the mean value $\tilde{z}_{i}{ }^{(q)}$ is a vector made of the respective probabilities to be in each of the $K$ classes. In the simulated field algorithm, $\tilde{z}_{i}^{(q)}$ is simulated from $P\left(z_{i} \mid \mathbf{y}, \tilde{z}_{\mathcal{N}(i)}^{(q)} ; \Psi^{(q-1)}\right)$.

In both cases, the approximate EM decomposes into the following $\mathrm{E}$ and $\mathrm{M}$ steps:

The E-step consists of computing, using equation (A.2), the approximate posteriors denoted by $\tilde{t}_{i k}^{(q)}$ : for all $i$ and $k, \quad \tilde{t}_{i k}^{(q)}=P\left(Z_{i}=e_{k} \mid \mathbf{y}, \tilde{z}_{\mathcal{N}(i)}^{(q)} ; \Psi^{(q-1)}\right)$, while the M-step consists of updating the parameters. The risk updates are available in closed-form (equation (A.3)) while the MRF prior parameters need to be computed numerically (equation (A.4)):

$$
\text { for all } k, \quad \lambda_{k}^{(q)}=\frac{\sum_{i \in S} \tilde{t}_{i k}^{(q)} y_{i}}{\sum_{i \in S} n_{i} y_{i}},
$$




$$
\text { and } \beta^{(q)}=\arg \max _{\beta} \sum_{i \in S} \sum_{k=1}^{K} \tilde{t}_{i k}^{(q)} \log \tilde{\pi}_{i k}^{(q)}(\beta) \text {, }
$$

where $\tilde{\pi}_{i k}^{(q)}(\beta)=P\left(Z_{i}=e_{k} \mid \tilde{z}_{\mathcal{N}(i)}^{(q)} ; \beta\right)$. Computing the gradient in (A.4) leads to further formulas given in Section A.2.

Using the variational approximations mentioned in the paper leads to algorithms which in their general form consist of repeating the following two steps. At iteration $q$,

(1) Create from the data $\mathbf{y}$ and some current parameter estimates $\Psi^{(q-1)}$ a configuration $\tilde{z}^{(q)}=$ $\left\{\tilde{z}_{1}^{(q)}, \ldots, \tilde{z}_{N}^{(q)}\right\}$, i.e. values for the $Z_{i}$ 's that satisfy some consistency conditions [see 1]. Replace the Markov distribution $P(\mathbf{z} ; \beta)$ by the factorized distribution $\prod_{i \in S} P\left(z_{i} \mid \tilde{z}_{\mathcal{N}(i)}^{(q)} ; \beta\right)$. It follows that the joint distribution $P(\mathbf{y}, \mathbf{z} ; \Psi)$ can also be approximated by a factorized distribution:

$$
\prod_{i \in S} \mathcal{P}\left(y_{i} ; n_{i} z_{i}^{t} \lambda\right) P\left(z_{i} \mid \tilde{z}_{\mathcal{N}(i)}^{(q)} ; \beta\right)
$$

and the two problems encountered when considering the EM algorithm with the exact joint distribution disappear. The second step is therefore,

(2) Apply the EM algorithm for this factorized model with starting values $\Psi^{(q-1)}$, to get updated estimates $\Psi^{(q)}$ of the parameters.

Note also that to save additional notation, the updating described above is synchronous while we actually implemented a sequential updating of the $\tilde{z}_{i}{ }^{(q)}$ 's: each site $i$ is updated in turn using the new values of the other sites as soon as they become available rather than waiting until all sites have been updated. Step (1) can then be iterated a number of times to guarantee that the corresponding consistency equations are satisfied. In practice the fixed point is reached in few iterations. Similarly, in step (2), performing a small number of EM iterations is usually enough.

\section{A.2 Details on the M-step}

The HMRF estimation provides us with estimations for the risk values $\lambda_{k}$, for $k=1, \ldots, K$, but also for the hidden field parameters, i.e matrix $\mathbb{B}$ and vector $\alpha$.

Computing the gradient in equation (A.4) with $\beta=(\alpha, \mathbb{B})$ and $\mathbb{B}$ of the form specified in the introduction above, the solutions $\alpha$ and $b$ of equation (A.4) satisfy:

$$
\begin{gathered}
\text { for } k=1, \ldots, K, \quad \sum_{i \in S} \tilde{\pi}_{i k}^{(q)}(\alpha, b)=\sum_{i \in S} \tilde{t}_{i k}^{(q)} \\
\text { and } \sum_{i \in S} \sum_{k=1}^{K}\left(\tilde{t}_{i k}^{(q)}-\tilde{\pi}_{i k}^{(q)}(\alpha, b)\right)\left(\sum_{j \in \mathcal{N}(i)} \tilde{z}_{j}^{(q)}(k)+\frac{1}{2}\left(\tilde{z}_{j}^{(q)}(k-1)+\tilde{z}_{j}^{(q)}(k+1)\right)\right)=0
\end{gathered}
$$




\section{B A search procedure for initializing EM}

\section{B.1 Illustration of the initialization strategy for Poisson mixtures}

To illustrate the proposed strategy and the differences with other initializations using random parameter values or random partitions, we consider a simple non spatial two-class case where the true parameter values are $\lambda_{1}=0.1, \lambda_{2}=0.2$ and the proportions for the two classes are $\pi_{1}=\pi_{2}=0.5$. We consider a hundred of sites $N=100$ and create values for the population sizes $n_{i}$ by sampling at random with replication among the integers between 10 and 109 . We then simulate the $y_{i}$ 's from a two component Poisson mixture model using equation (3) in the paper. The histogram of the $y_{i}$ 's is shown in Supplementary Figure 1 (a). The corresponding $\bar{\lambda}$ value is around 0.141 .

In the two-class case, it follows from equation (5) in the paper that the EM trajectories and therefore the maximum likelihood estimates of $\lambda_{1}$ and $\lambda_{2}$ are constrained to live in the greyed area of Supplementary Figure 1 (b) delimited by vertical and horizontal lines $\lambda_{1}=\bar{\lambda}$ and $\lambda_{2}=\bar{\lambda}$. The latter Supplementary Figure also shows 100 initial values of $\lambda_{1}$ and $\lambda_{2}$ drawn uniformly at random between 0 and 0.4 and ordered so that $\lambda_{1}<\lambda_{2}$. Among the 100 points, only 42 lie within the EM trajectory area. The point corresponding to the true values $\lambda_{1}=0.1$ and $\lambda_{2}=0.2$ is marked with an "X". For comparison, we show respectively in Supplementary Figures 1 (c) and (d), 100 values obtained by using our strategy and starting from 100 random partitions. As expected, random partition initializations tend to produce values close to $\bar{\lambda}$ and fail in exploring the space efficiently. In contrast, our strategy better explores the grey area and in particular around the true values $(0.1,0.2)$. The phenomenon is even more striking as we increase the number of simulated values for initialization.

In Supplementary Figure 1 (e), (f) and (g), we then show the $\left(\lambda_{1}, \lambda_{2}\right)$ values obtained after 1 iteration of EM starting from the initial values obtained with the three initializing strategies. To better visualize the differences on the plots, we show only 20 such values. They lie all within the grey area but only our proposed strategy tends to produce values near the true values. Random partition values are somewhat clustered at the wrong place and values from random parameter values are more dispersed. Among the 20 points, more points are likely not to lead close to the desired solution.

\section{B.2 A full search procedure}

To complete our Search procedure, we need in addition to initial $\lambda$ values, starting positions for the Markov prior parameters $\beta=(\alpha, \mathbb{B})$. When $\mathbb{B}$ reduces to some value $b$, a typical sequence of $\lambda$ and $b$ values obtained using our full search procedure is shown in Supplementary Figure 2 for a realistic example closed to the real data considered in the paper. In this Supplementary Figure, we show also the parameters values obtained with the same initialization (resulting from Search 1) but skipping the Search 2 stage and running directly our EM algorithm letting all parameters free. We observe clearly that without the Search 2 stage, the $b$ parameter is rapidly increasing to very high values which tends to trap the algorithm into some meaningless solution with a too high spatial interaction. Also the $\alpha$ values not shown here stay between -4 


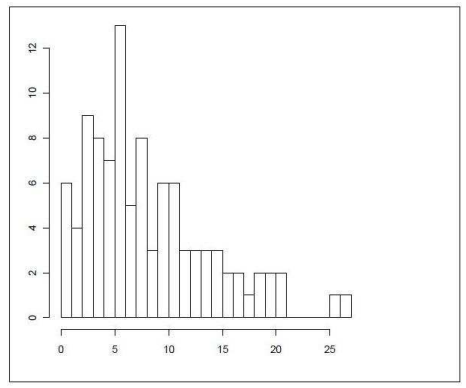

(a)

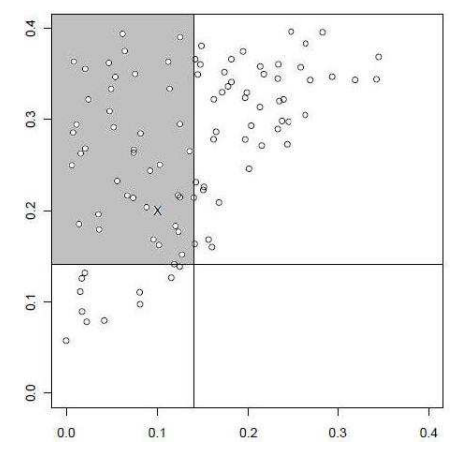

(b)

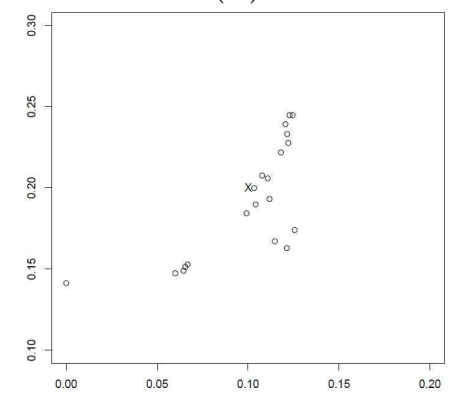

(e)

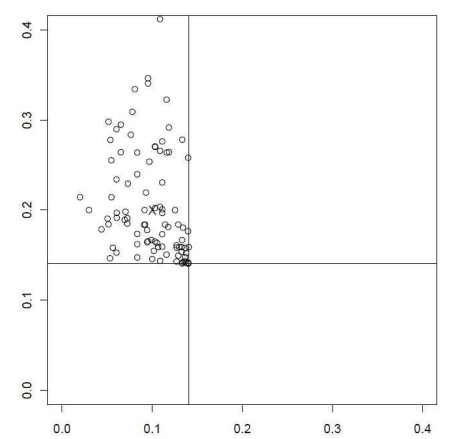

(c)

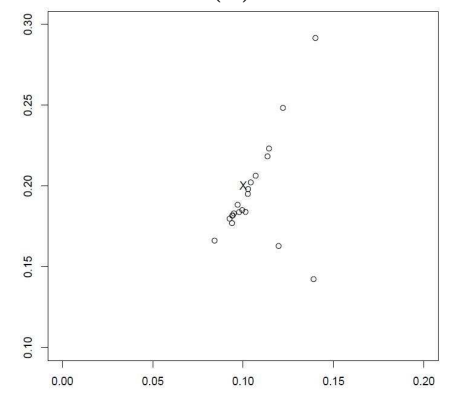

(f)

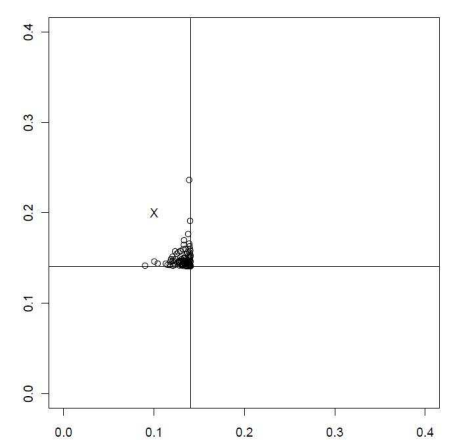

(d)

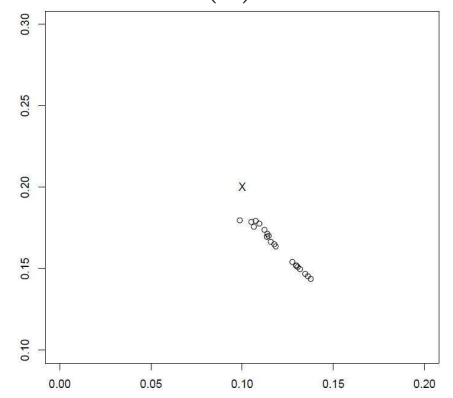

(g)

Supplementary Figure 1: Initialization strategies for a two-class Poisson mixture, a non spatial example. Figure (a): histogram of 100 values from our model with $\lambda_{1}=0.1$, $\lambda_{2}=0.2, b=0$ and $\alpha$ is so that the two classes are in equal proportion; (b): 100 values of $\left(\lambda_{1}, \lambda_{2}\right)$ sampled at random between 0 and 0.4 and ordered so that $\lambda_{1}<\lambda_{2}$; (c): 100 values of $\left(\lambda_{1}, \lambda_{2}\right)$ generated from the strategy using the EM trajectories property and $(\mathrm{d})$ : 100 values of $\left(\lambda_{1}, \lambda_{2}\right)$ generated from random partitions of the simulated data. The true values $(0.1,0.2)$ are indicated by a "X". (e): 20 values of $\left(\lambda_{1}, \lambda_{2}\right)$ after 1 iteration of EM when the initial values are random, (f): when the initial values are generated using the proposed strategy and (g): when the initial values are obtained from random partitions. 
and 1 when Search 2 is included while in the other case, the $\alpha(1)$ value is decreasing rapidly to a very low negative value as a compensation for the corresponding high value of $b$. Note that this pathological behavior is due to the very low risk values used in this example, which can be observed in animal epidemiology.

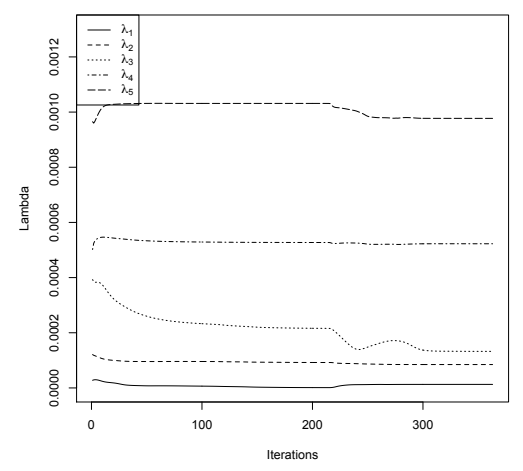

(a)

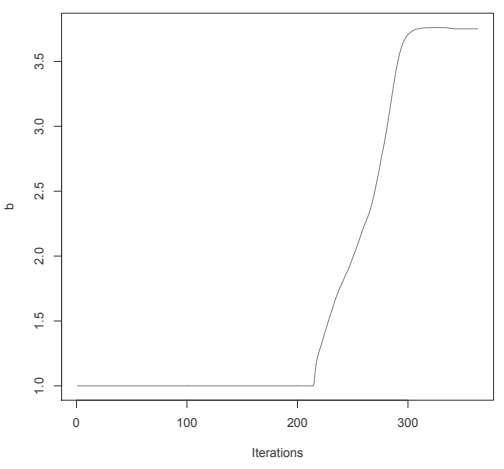

(c)

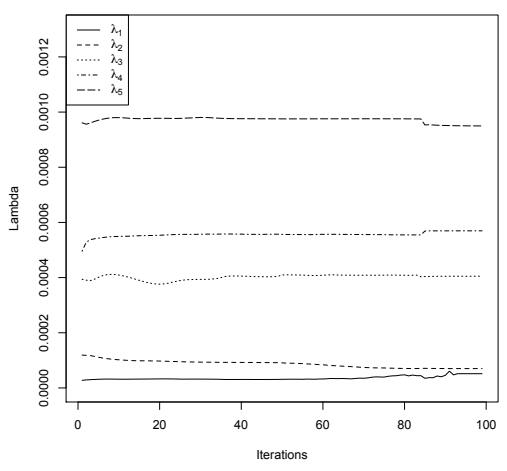

(b)

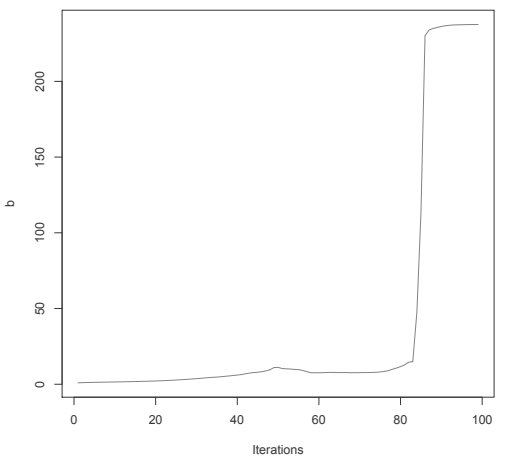

(d)

Supplementary Figure 2: A full search procedure. Parameters obtained for a simulated data example of a hidden Markov model with 5 mixture components. (a): $\lambda$ values obtained using our full search procedure. (b): $\lambda$ values obtained skipping the Search 2 step. (c): spatial interaction parameter $b$ value obtained using our full search procedure . (d): $b$ value obtained skipping the Search 2 step. Note the much larger scale in the latter plot.

\section{Illustration on simulated data sets}

To complete the typical simulated examples illustrated in the main manuscript, we considered other cases to further test the robustness of our model. In subsection C.1 we report results for a data set with a North-South gradient corresponding to risks smoothly (linearly) decreasing 
from North to South from $10^{-3}$ to $10^{-5}$. This corresponds to a case more favorable to the BYM model and puts our model at comparative disadvantage. In subsection C.2, to illustrate the robustness of our model to non smooth risk level gradation, we considered two additional simulations using the same synthetic risk partitions (Figure 1 (b,c) in the main paper) but with permuted risk levels. In addition, to further investigate the algorithms, we repeated the simulations a hundred times with the same true risk values (see subsection C.3).

\section{C.1 A North-South gradient example}

The true risks range from $10^{-5}$ to $10^{-3}$ (Supplementary Figure 3 (a)) but the estimated risks for all models remain between $10^{-4}$ and $10^{-3}$. The high risk regions are relatively well identified while the very low risk ones $\left(10^{-5}\right)$ are not recovered with a minimum risk level estimated at $1.5310^{-4}$ (Supplementary Figure $3(\mathrm{k})$ ).

Regarding high risk regions, our model outperforms the Potts model in providing larger risk ranges with the highest risk level estimated at $9.3110^{-4}$ (Supplementary Figure $3(\mathrm{j})$ ). Note that in Supplementary Figure $3(\mathrm{k})$ (Potts model) for $K=7$, the risk classes corresponding to $\lambda_{7}=9.9410^{-4}$ and $\lambda_{5}=0$ are lost in that no regions are assigned to these levels in the mapping (the $\lambda_{5}$ value being badly estimated). Similarly for our model (Supplementary Figure $3(\mathrm{j})$ ), one risk class $\left(\lambda_{7}=5.8810^{-4}\right)$ is confined to the North of the Corsica highland suggesting a potential problem with this estimation. Overall, we observe that both models have difficulty in finding $K=7$ classes in the observed data. As a matter of fact, using BIC (see Supplementary Table 1), the selected $K$ is 5. By comparison, the BYM model is doing well in estimating very high risk regions in the North but wrongly classifies as high risk too regions in the South-East. This may be again due to the presence there of low population sizes.

In terms of shape of the identified regions, the Potts and BYM models sometimes provide less satisfying partitions (see e.g Supplementary Figure $3(\mathrm{~h})$ ). Although in this continuous risk example, no true partition is available, because of the North-South gradient we expect horizontal risk classes.

\begin{tabular}{|l|c|c|}
\hline$K$ & Potts & Our model \\
\hline $\mathbf{2}$ & 4196.6 & 4196.6 \\
\hline $\mathbf{3}$ & 4029.4 & 4023.5 \\
\hline $\mathbf{4}$ & 4051.3 & 4028.9 \\
\hline $\mathbf{5}$ & $\mathbf{4 0 1 9 . 8}$ & $\mathbf{4 0 1 2 . 9}$ \\
\hline $\mathbf{6}$ & 4027.5 & 4013.0 \\
\hline $\mathbf{7}$ & 4055.7 & 4036.9 \\
\hline
\end{tabular}

Supplementary Table 1: BIC values for the Potts and our models for $K=2$ to 7 in the North-South gradient data set case. The selected $K$ is indicated with bold characters. 


\section{C.2 Abrupt transitions with 3 and 5-classes}

To create abrupt transitions, we permuted the risk levels from the previous synthetic risk partitions (Figure 1 (b,c) in the main paper). In the 3-class example, $\lambda_{1}=1 \times 10^{-5}, \lambda_{2}=$ $1 \times 10^{-3}$ and $\lambda_{3}=1 \times 10^{-4}$ so that we locate now the highest risk next to the lowest one. In the 5-class case, similarly the risk levels are permuted to $\lambda_{1}=5 \times 10^{-5}, \lambda_{2}=5 \times 10^{-4}$, $\lambda_{3}=1 \times 10^{-4}, \lambda_{4}=1 \times 10^{-5}$ and $\lambda_{5}=1 \times 10^{-3}$. Results obtained with the BYM model and both the Potts and our models are shown in Supplementary Figure 4. The Potts and our model provide similar results (see also the Dice scores in Supplementary Table 2) both more satisfying than BYM. In the 3-class example, the particularity of our model appears clearly at the border of two of the classes with some wrongly classified hexagons. Paradoxically, overall the HMRF model performance is better than in the smooth gradation case. The fact that neighboring risk levels are now more different induces better separated classes and makes the classification easier.

\begin{tabular}{|l|l|l|l|l|l|}
\hline \multicolumn{7}{|c|}{ Three-class data set } \\
\hline Our model & 0.989 & 0.990 & 0.997 & & \\
Potts & 0.998 & 0.998 & 0.997 & & \\
\hline \multicolumn{7}{|c|}{ Five-class data set } \\
\hline Our model & 0.760 & 0.662 & 0.030 & 0.700 & 0.818 \\
Potts & 0.749 & 0.371 & 0.718 & 0.762 & 0.832 \\
\hline
\end{tabular}

Supplementary Table 2: Dice Scores for the Potts and our model for the $K=3$ and $K=5$ examples in the abrupt transition case.

\section{C.3 Boxplots of the risk parameters over 100 runs}

For the true values of $K$, the performance is then evaluated considering both average classification performance and risk value estimation. The boxplots obtained for the risk parameters over 100 runs are shown below. For the 3-class example, the average estimation of the risk values is in general close to the real parameter values for the three strategies. However, $S_{E M M}$ tends to overestimate low risks. $S_{t r a}$ and $S_{\text {rand }}$ give similar results. In terms of DSC values, $S_{\text {rand }}$ outperforms $S_{t r a}$ on average and shows smaller variances. However the boxplots of Supplementary Figure 5 (a-c) show that the median risk values are very close for both strategies. In the 5-class case, $S_{\text {tra }}$ provides better average risks for the high and very high risk values. In terms of DSC values, the average values are higher and variances generally lower for $S_{t r a}$. For estimated risks, this is also the case for medium to very high risks. The $S_{t r a}$ strategy seems to provide better and more stable results for higher risk values, which is a desirable feature in epidemiology. The boxplots in Supplementary Figure $5(\mathrm{~d}-\mathrm{h})$ below show that this is generally compensated by a worse estimation of the medium risk class compared to $S_{\text {rand }}$ (Supplementary Figure 5 (f)).

For the 3 -class case, we can notice that the estimator $\hat{\lambda}$ predicts observations of the parameter $\lambda$ with a good accuracy. However, the estimation of parameters associated with the highest 
risk region is much more precise than for the lowest risk. For the 5-class case, larger variations are observed for the class which in general disappears $\left(\lambda_{3}\right)$. The estimation is also more precise for higher risks than for the lower ones.

\section{The Bovine Spongiform Encephalopathy data set}

The Bovine Spongiform Encephalopathy (BSE) is a non contagious neurodegenerative disease in cattle. This sudden and unexpected disease threatened bovine production in Europe and has been intensively studied, in particular for spatial patterns. It is transmitted by meat and bone meal. Since there is no direct transmission and no vector, spatial analysis is important to understand and explain the geographical localisation of the cases. In our data set, the numbers of observed cases are available for each hexagonal geographical unit in France. These cases occurred between July 1,2001 and December 31,2005 although at that time meat and bone meal had been already forbidden for cattle in France.

We first apply our model initialized with the $S_{t r a}$ strategy described in the main paper. Regarding the number of classes, the approximated BIC of [3] indicates $K=3$. Using the same initialization and computing BIC, we selected $K=3$ for both the standard Potts model and smooth gradation model equivalent to our model in this case. Comparing all BIC values (See Supplementary Table 3), the best scores for these two models and ours are equivalent but the risk map provided by the smooth gradation and our models (Figure 4 (c) in the main paper) makes clearly more sense than the one obtained with the standard Potts model (see Figure $4(\mathrm{~g})$ in the main paper).

\begin{tabular}{|l|c|c|c|}
\hline$K$ & Potts & smooth gradation & Our model \\
\hline $\mathbf{2}$ & 1597.2 & 1597.2 & 1607.2 \\
\hline $\mathbf{3}$ & $\mathbf{1 5 8 5 . 9}$ & $\mathbf{1 5 8 8 . 6}$ & $\mathbf{1 5 8 8 . 6}$ \\
\hline $\mathbf{4}$ & 1598.8 & 1597.2 & 1598.0 \\
\hline $\mathbf{5}$ & 1593.5 & 1606.7 & 1595.9 \\
\hline $\mathbf{6}$ & 1600.2 & 1607.8 & 1602.0 \\
\hline
\end{tabular}

Supplementary Table 3: BIC values for the Potts, smooth gradation and our models for $K=2$ to 6 in the BSE data set case. The selected $K$ is indicated with bold characters. 


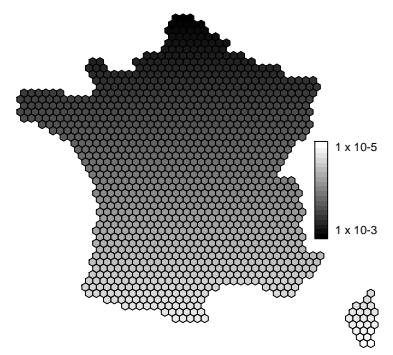

(a)

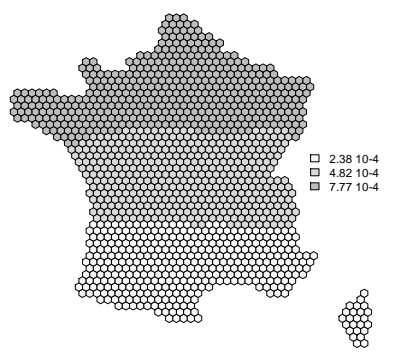

(d)

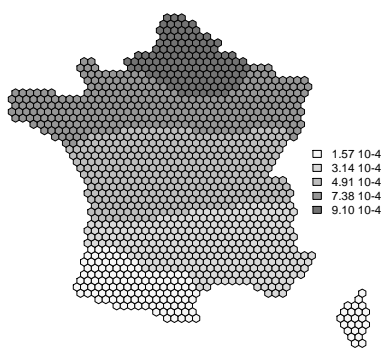

(g)

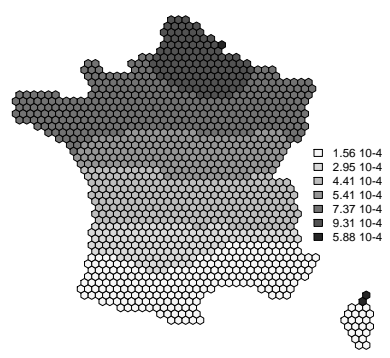

(j)

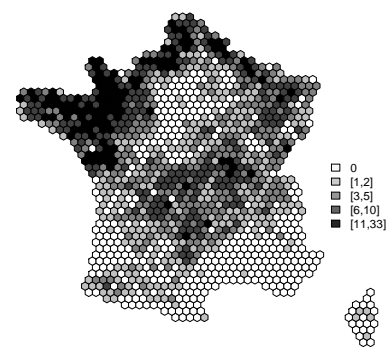

(b)

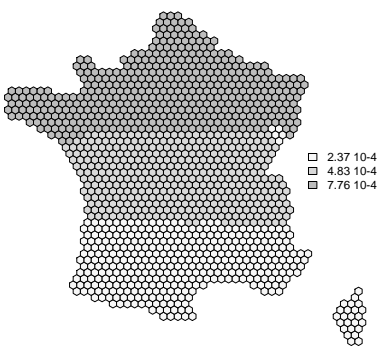

(e)

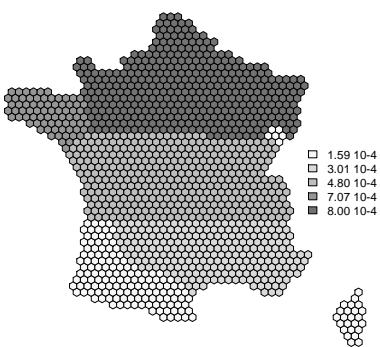

(h)

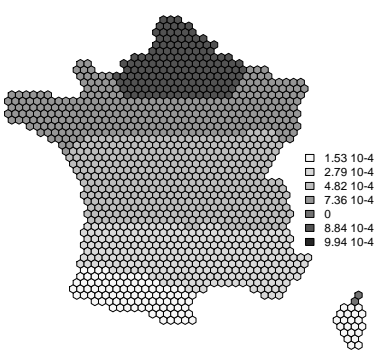

(k)

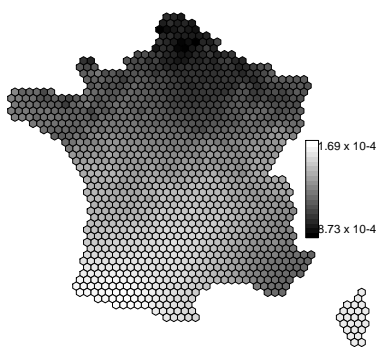

(c)

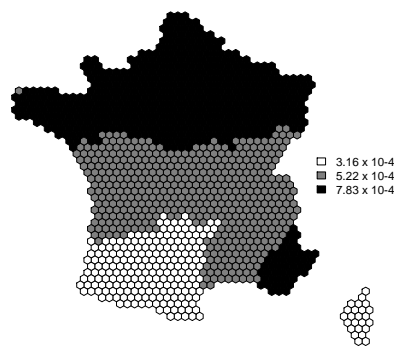

(f)

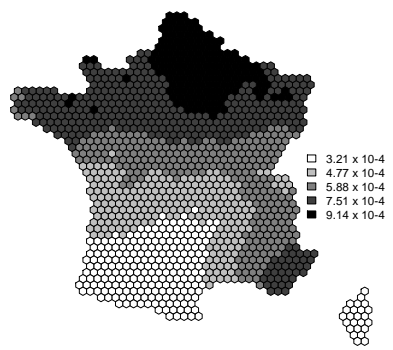

(i)

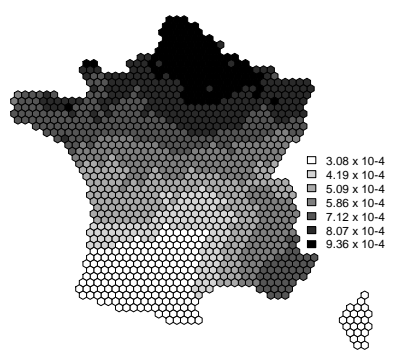

(1)

Supplementary Figure 3: North-South gradient. (a) True risk map, (b) Simulated counts using the cattle population of Figure 1(a) in the paper and (c) Continuous risk map from the BYM model. Estimated risk maps for resp. $K=3,5,7$ with $(\mathrm{d}, \mathrm{g}, \mathrm{j})$ our model, $(\mathrm{e}, \mathrm{h}, \mathrm{k})$ the Potts model and (f,i,l) the BYM model. The selected number of classes is $K=3$ (see Table 1 ). 


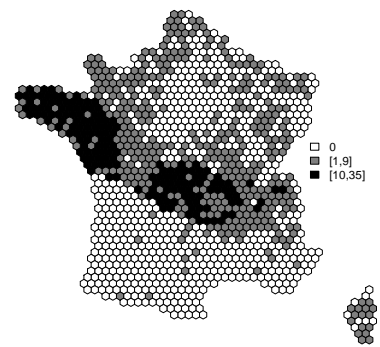

(a)

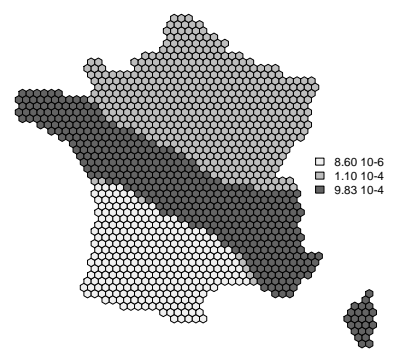

(c)

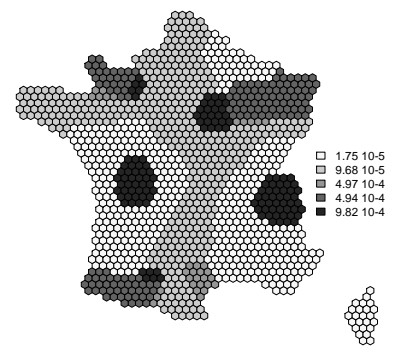

(f)

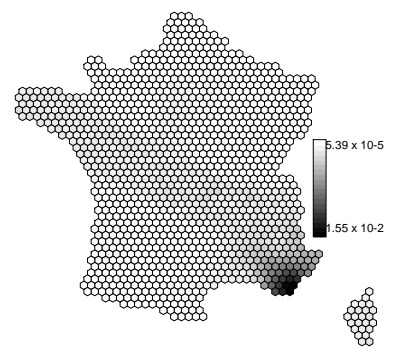

(i)

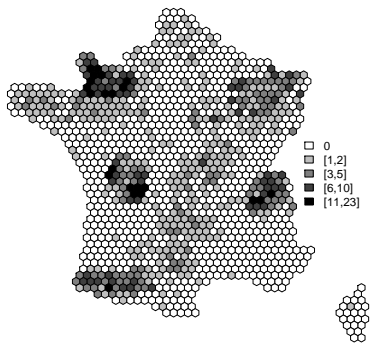

(b)

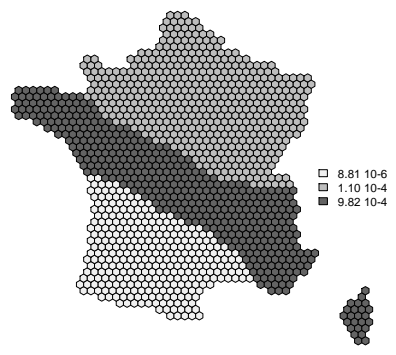

(d)

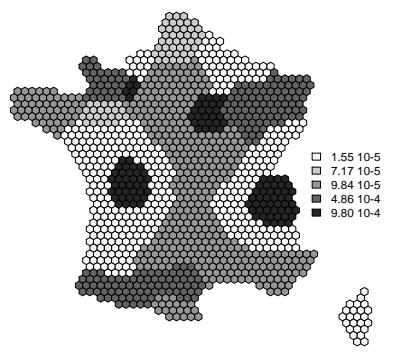

(g)

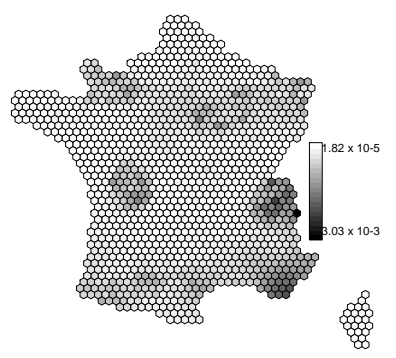

(j)

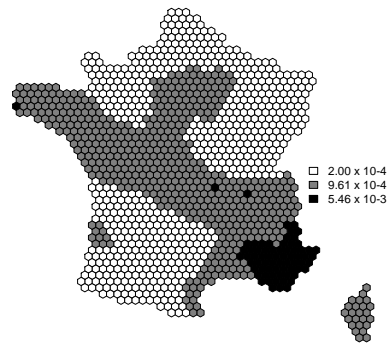

(e)

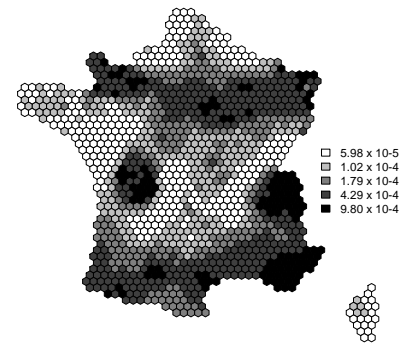

(h)

Supplementary Figure 4: 3 and 5 class examples with abrupt risk transitions. (a,b) Simulated counts using the cattle population of Figure 1(a) in the paper. Estimated risk maps for resp. $K=3$ and 5 with (c,f) our model, $(\mathrm{d}, \mathrm{g})$ the Potts model and (e,h) the BYM model. Continuous risk maps from the BYM model are shown in $(\mathrm{i}, \mathrm{j})$. 


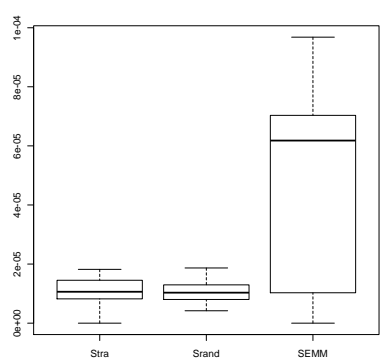

(a): $\lambda_{1}$ (low)

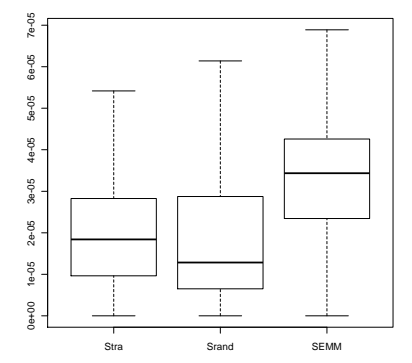

(d): $\lambda_{1}$ (very low)

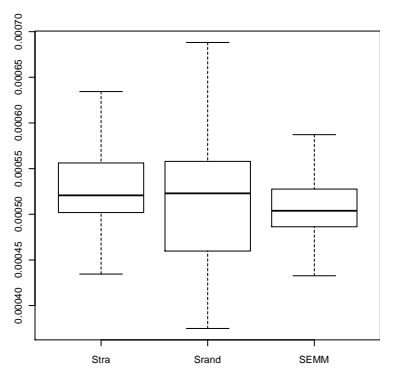

(g): $\lambda_{4}$ (high)

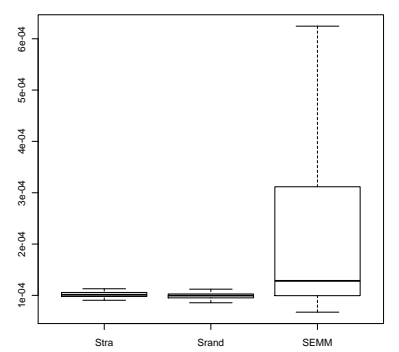

(b): $\lambda_{2}$ (medium)

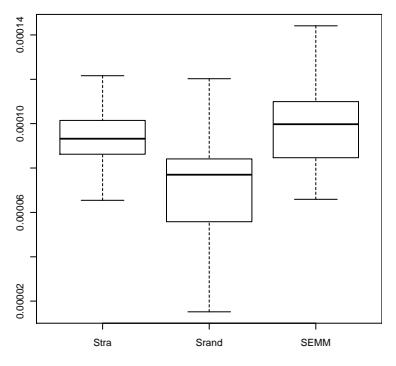

(e): $\lambda_{2}$ (low)

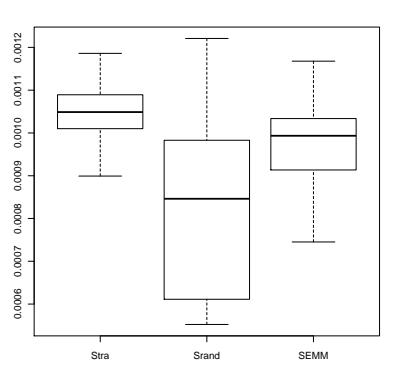

(h): $\lambda_{5}$ (very high)

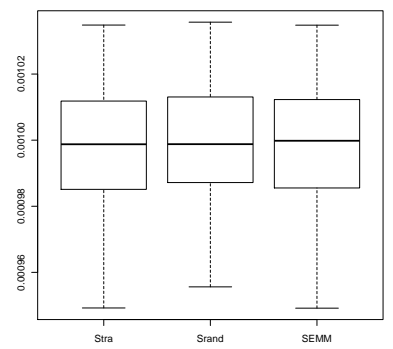

(c): $\lambda_{3}$ (high)

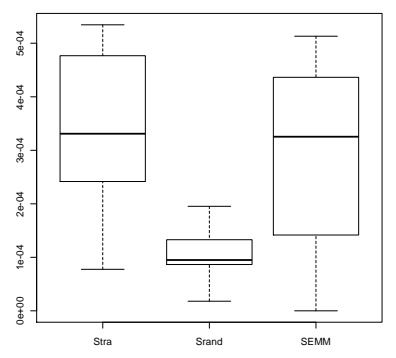

(f): $\lambda_{3}$ (medium)

Supplementary Figure 5: Risk parameters over 100 runs for the Stra, Srand and SEMM initializations. (a-c): 3-class example . (d-h): 5-class example. Outliers have been removed for the plots. 


\section{References}

[1] G. Celeux, F. Forbes, and N. Peyrard. EM procedures using mean field-like approximations for Markov model-based image segmentation. Pattern Recognition, 36:131-144, 2003.

[2] A.P. Dempster, N.M. Laird, and D.B. Rubin. Maximum likelihood from incomplete data via the EM algorithm. Journal of the Royal Statistical Society: Series B (Methodological), 39(1):1-38, 1977.

[3] F. Forbes and N. Peyrard. Hidden Markov model selection based on mean field like approximations. IEEE Trans. on Pattern Analysis and Machine Intelligence, 25(8):1089-1101, 2003. 